PROCEEDINGS OF THE

AMERICAN MATHEMATICAL SOCIETY

Volume 128, Number 9, Pages 2675-2682

S 0002-9939(00)05564-7

Article electronically published on February 28, 2000

\title{
DIAGONAL DOMINANCE AND HARMLESS OFF-DIAGONAL DELAYS
}

\author{
JOSEF HOFBAUER AND JOSEPH W.-H. SO
}

(Communicated by Hal L. Smith)

\begin{abstract}
Systems of linear differential equations with constant coefficients, as well as Lotka-Volterra equations, with delays in the off-diagonal terms are considered. Such systems are shown to be asymptotically stable for any choice of delays if and only if the matrix has a negative weakly dominant diagonal.
\end{abstract}

\section{INTRODUCTION}

Consider a system of retarded linear differential equations with constant coefficients of the form

$$
\dot{x}_{i}=\sum_{j=1}^{n} a_{i j} x_{j}\left(t-\tau_{i j}\right), \quad \text { for } i=1, \ldots, n,
$$

with

$$
\tau_{i j} \geq 0 \quad \text { for } 1 \leq i \neq j \leq n \quad \text { and } \quad \tau_{i i}=0 \quad \text { for } i=1, \ldots, n .
$$

This paper deals with the following question:

(*) For which matrices $A=\left(a_{i j}\right)$ is the trivial solution $x=0$ of (1.1) asymptotically stable for any choice of delays satisfying (1.2)?

Such type of stability has been referred to as 'absolute stability' in the literature (cf. El'sgol'ts and Norkin [3, p. 175]). In the context of population modelling, the term 'harmless' delays has also been used (see Gopalsamy [5]). For $n=2$, this question was answered by Lu and Wang [12]. They showed that $(*)$ holds iff $a_{11}, a_{22}<0$ and $a_{11} a_{22}>\left|a_{12} a_{21}\right|$ (with equality allowed in the latter if $a_{12} a_{21}<0$ ). Actually, their result was for Lotka-Volterra equations but the proof applies to (1.1) with minor modifications. We extend this result to arbitrary $n$, both for the linear case (1.1) and the Lotka-Volterra case (3.1).

Definition. Let $\tilde{A}=\left(\tilde{a}_{i j}\right)$ be the matrix with entries $\tilde{a}_{i i}=a_{i i}$ and $\tilde{a}_{i j}=\left|a_{i j}\right|$ for $i \neq j$. $A$ is said to be weakly diagonally dominant if all the principal minors of $-\tilde{A}$ are non-negative.

Received by the editors October 23, 1998.

1991 Mathematics Subject Classification. Primary 34K20.

This research was partially supported by NSERC of Canada, grant number OGP36475.

(C)2000 American Mathematical Society 
Our main result is the following.

Theorem 1. (1.1) is asymptotically stable for all choices of delays of the form (1.2) if and only if $a_{i i}<0$ for $i=1, \ldots, n$, $\operatorname{det} A \neq 0$ and $A$ is weakly diagonally dominant.

For quasimonotone matrices $A$ (i.e., $a_{i j} \geq 0$ for $i \neq j$ ), a similar result was obtained by Győri (1992).

\section{Proof of Theorem 1}

The characteristic equation of the delay differential equation (1.1) is given by

$$
\operatorname{det}\left(\begin{array}{ccc}
a_{11}-\lambda & a_{12} e^{-\lambda \tau_{12}} & \cdots \\
a_{21} e^{-\lambda \tau_{21}} & a_{22}-\lambda & \cdots \\
& \cdots &
\end{array}\right)=0 .
$$

Since $x(t)=c e^{\lambda t}$ is a solution of (1.1) for suitable $c \neq 0$ iff $\lambda$ satisfies (2.1), by Corollary 6.1 of Hale and Verduyn Lunel [7, p. 215], the trivial solution $x=0$ of (1.1) is asymptotically stable iff all the roots of (2.1) have negative real part.

\subsection{Sufficiency part.}

Lemma 1. If $A$ is weakly diagonally dominant, then all roots of (2.1) have negative real part, with the possible exception of $\lambda=0$.

Proof. We first consider irreducible matrices $A$. By Theorem 5.9 of Fiedler [4] p. 124], for an irreducible weakly diagonally dominant matrix $A$, there is a $c>0$ such that $\tilde{A} c \leq 0$, i.e., there exist $c_{i}>0$ such that

$$
a_{i i} c_{i}+\sum_{j \neq i}\left|a_{i j}\right| c_{j} \leq 0 \quad \text { for all } i=1, \ldots, n .
$$

Suppose, for some set of delays $\tau_{i j}$ satisfying (1.2), there exists a root $\lambda$ of (2.1) with $\operatorname{Re} \lambda \geq 0$. Then $\lambda$ is an eigenvalue of the matrix $B=\left(b_{i j}\right)$, where $b_{i j}=a_{i j} e^{-\lambda \tau_{i j}}$. Since $b_{i i}=a_{i i} \leq 0$ and $\left|b_{i j}\right| \leq\left|a_{i j}\right|$, (2.2) implies

$$
b_{i i} c_{i}+\sum_{j \neq i}\left|b_{i j}\right| c_{j} \leq 0 \quad \text { for } i=1, \ldots, n .
$$

Applying Geršgorin's theorem (cf. Lancaster and Tismenetsky [10, p. 371]) to the matrix $\hat{B}=\left(c_{i}^{-1} b_{i j} c_{j}\right)$, which is similar to $B$, we know that the eigenvalue $\lambda$ of $\hat{B}$ is contained in a circle with center $b_{i i} \leq 0$ and radius at most $\left|b_{i i}\right|$ (for some $i$ ). Hence either $\operatorname{Re} \lambda<0$ or $\lambda=0$.

In the case of a reducible matrix $A$, we can (by suitably relabeling the indices) turn $A$ into an upper block triangular matrix with irreducible (or zero) blocks along the diagonal (cf. (3.6) of Berman and Plemmons [2, p. 39]). Since index relabeling is done via a permutation matrix and does not affect the principal minors of $A$, each diagonal block is itself weakly diagonally dominant. The result now follows by applying the previous argument to each irreducible diagonal block.

The solution $\lambda=0$ of $(2.1)$ is possible only if $\operatorname{det} A=0$ which is excluded. This concludes the proof of the sufficiency part of Theorem 1. 
2.2. Necessity part. We start with three lemmas.

Lemma 2. If $a_{i i}<0$ for all $i=1, \ldots, n$ and $\operatorname{det}(-\tilde{A})<0$, then there exist delays $\tau_{i j}$ satisfying (1.2) such that (2.1) has a root $\lambda$ with $\operatorname{Re} \lambda>0$.

Proof. Consider the function

$$
F_{\varepsilon}(z)=\operatorname{det}\left(\begin{array}{ccc}
a_{11}-z \varepsilon & a_{12} e^{-z \eta_{12}} & \cdots \\
a_{21} e^{-z \eta_{21}} & a_{22}-z \varepsilon & \cdots \\
& \cdots &
\end{array}\right)
$$

where

$$
\eta_{i j}=\left\{\begin{array}{l}
\frac{1}{2}, \quad \text { for } a_{i j}<0, \\
1, \quad \text { for } a_{i j} \geq 0 .
\end{array}\right.
$$

For $z=x+2 \pi i$, where $x$ is real, $F_{0}(z)$ becomes

$$
D(x)=\operatorname{det}\left(\begin{array}{ccc}
a_{11} & \left|a_{12}\right| e^{-x \eta_{12}} & \ldots \\
\left|a_{21}\right| e^{-x \eta_{21}} & a_{22} & \cdots \\
& \cdots &
\end{array}\right) .
$$

By assumption, $(-1)^{n} D(0)=\operatorname{det}(-\tilde{A})<0$, and $(-1)^{n} D(\infty)=(-1)^{n} a_{11} \ldots a_{n n}$ $>0$. Hence, by intermediate value theorem, there exists $\hat{x}>0$ such that $D(\hat{x})=0$ and $\hat{z}=\hat{x}+2 \pi i$ is a zero of $F_{0}$. It follows from Rouché's theorem (cf. Ahlfors [1, p. 153]) that the analytic function $F_{\varepsilon}$ has a zero $\hat{z}(\varepsilon)$ near $\hat{z}$ for small $\varepsilon>0$. Clearly, $\lambda=\hat{z}(\varepsilon) \varepsilon$ and $\tau_{i j}=\frac{\eta_{i j}}{\varepsilon}$ satisfy (2.1), with $\operatorname{Re} \lambda>0$.

Lemma 3. Let $f(\lambda)$ be a real polynomial of degree $n$ with $n$ real zeros, including 0 . Then the equation

$$
f(\lambda)=e^{-\lambda \tau}
$$

has a solution $\lambda$ with $\operatorname{Re} \lambda>0$ for some $\tau>0$.

Proof. Write $f(\lambda)$ as $\alpha \prod_{i=1}^{n}\left(\lambda-\lambda_{i}\right)$, where $0 \neq \alpha \in \mathbb{R}$ and $\lambda_{i} \in \mathbb{R}$ with $\lambda_{1}=0$. We first show (2.3) has a purely imaginary root $\lambda=i \omega$, where $\omega>0$, for some $\tau>0$. By suitably choosing $\tau>0, f(i \omega)=e^{-i \omega \tau}$ holds iff $|f(i \omega)|=1$. Since $|f(0)|=0$ and $\lim _{\omega \rightarrow \infty}|f(i \omega)|=\infty$, the existence of $\omega>0$ follows from the intermediate value theorem. Let $f(\hat{\lambda})=e^{-\hat{\lambda} \hat{\tau}}$, where $\hat{\lambda}=i \hat{\omega}$ and $\hat{\omega}, \hat{\tau}>0$.

Next, we show that by slightly increasing $\tau$ past $\hat{\tau}$, the solution $\lambda$ crosses the imaginary axis. Indeed, let $g(\lambda, \tau)=f(\lambda)-e^{-\lambda \tau}$. Then

$$
g_{\lambda}(\hat{\lambda}, \hat{\tau})=e^{-\hat{\lambda} \hat{\tau}}\left[\sum_{i=1}^{n} \frac{1}{\hat{\lambda}-\lambda_{i}}+\hat{\tau}\right] \neq 0
$$

since

$$
\operatorname{Im}\left(\sum_{i=1}^{n} \frac{1}{\hat{\lambda}-\lambda_{i}}\right)=-\hat{\omega} \sum_{i=1}^{n} \frac{1}{\lambda_{i}^{2}+\hat{\omega}^{2}}<0 .
$$

The implicit function theorem applied to $(2.3)$ at $(\hat{\lambda}, \hat{\tau})$ yields the solution

$$
\lambda(\tau)=\hat{\lambda}+c(\tau-\hat{\tau})+O\left((\tau-\hat{\tau})^{2}\right)
$$

where $c=-\frac{g_{\tau}(\hat{\lambda}, \hat{\tau})}{g_{\lambda}(\hat{\lambda}, \hat{\tau})}$. It is easy to see that $\operatorname{Re} c>0$, and hence $\operatorname{Re} \lambda(\tau)>0$ for $\tau>\hat{\tau}$. 
Lemma 4. If $a_{i i}=0$ for some $i$ and $\operatorname{det} A \neq 0$, then there exist delays $\tau_{i j}$ satisfying (1.2) such that (2.1) has a root $\lambda$ with $\operatorname{Re} \lambda>0$.

Proof. Assume $a_{11}=0$. Since $\operatorname{det} A \neq 0$, there exists a non-zero term in the expansion of $\operatorname{det} A$, i.e. there is a permutation $\sigma$ of $\{1, \ldots, n\}$ such that $\prod_{i=1}^{n} a_{i \sigma_{i}} \neq$ 0 . The corresponding term in the expansion of (2.1) together with the product of the diagonal entries yields the 'truncated' equation

$$
\prod_{i=1}^{n}\left(a_{i i}-\lambda\right)+\operatorname{sgn}(\sigma) \prod_{i=1}^{n}\left(a_{i \sigma_{i}}-\lambda \delta_{i \sigma_{i}}\right) \cdot e^{-\lambda \sum_{i=1}^{n} \tau_{i \sigma_{i}}}=0 .
$$

After cancelling the factor $a_{i i}-\lambda$ (for each $i$ with $\sigma_{i}=i$ ), there remains an equation of the form (2.3), where $\tau=\sum_{i=1}^{n} \tau_{i \sigma_{i}}$. By Lemma 3, there is a solution $\hat{\lambda}$ of (2.5) with $\operatorname{Re} \hat{\lambda}>0$ for a suitable $\tau>0$. By letting $\tau_{i j} \rightarrow \infty$ whenever $j \neq i$ and $j \neq \sigma_{i}$, the remaining $n !-2$ terms in the expansion of (2.1) left out in (2.5) can be made arbitrarily small (in a fixed neighbourhood of $\hat{\lambda}$ ). Hence Rouché's theorem again shows the existence of a root $\lambda$ of (2.1) near $\hat{\lambda}$. This shows the lemma.

Returning to the proof of the sufficiency part, the asymptotic stability assumption on $x=0$ precludes $\lambda=0$ from being a root of (2.1). Hence $\operatorname{det} A \neq 0$.

If $a_{k k}>0$ for some $k$, then Rouché's theorem shows that (2.1) has a root near $a_{k k}$ for large $\tau_{i j}$. This contradiction together with Lemma 4 shows that $a_{i i}<0$ for all $i=1, \ldots, n$.

Now suppose that some principal minor of $-\tilde{A}$ is negative. Without loss of generality, we may assume $\operatorname{det}\left(-\tilde{A}_{k}\right)<0$, where $A_{k}=\left(a_{i j}\right)_{1 \leq i, j \leq k}$. Applying Lemma 2 to this principal submatrix yields a root $\hat{\lambda}$ with $\operatorname{Re} \hat{\lambda}>0$ for the characteristic equation (2.1) corresponding to $A_{k}$. By letting $\tau_{i j} \rightarrow \infty$ whenever $i>k$ or $j>k$, a similar perturbation argument as above shows that the full characteristic equation (2.1) (corresponding to $A$ ) has a root near $\hat{\lambda}$. In particular, (2.1) will have a root with positive real part, which contradicts the assumption of stability of $x=0$. This completes the proof of Theorem 1.

\section{LOtKa-Volterra EQUATIONS}

In this section we prove a similar result for a class of nonlinear differential equations, widely used in population dynamics, the Lotka-Volterra systems

$$
\dot{y}_{i}=y_{i}\left(r_{i}+\sum_{j=1}^{n} a_{i j} y_{j}\left(t-\tau_{i j}\right)\right), \quad i=1, \ldots, n .
$$

We assume in the following that there exists a positive vector $\hat{y}$ with

$$
r+A \hat{y}=0 .
$$

This $\hat{y}$ is then an equilibrium for (3.1), i.e., $y(t)=\hat{y}$ is a (constant) solution of (3.1).

We are interested in the absolute stability of $\hat{y}$; and we will show that this is the case under the same conditions on $A$ as in the linear case (1.1). That the strict version of diagonal dominance (all principal minors of $-\tilde{A}$ are positive) implies the global stability of the unique saturated equilibrium has been discussed in detail in Hofbauer and Sigmund [8] for ordinary Lotka-Volterra equations and in Kuang [9] for delayed versions like (3.1). That weak diagonal dominance is already sufficient 
is more subtle, and its proof uses ideas from $\mathrm{Lu}$ and Takeuchi [11, $\mathrm{Lu}$ and Wang [12] and Redheffer [13].

Theorem 2. Suppose there exists a positive vector $\hat{y}$ satisfying (3.2). Then $\hat{y}$ is globally asymptotically stable for (3.1) (for initial conditions $y_{i}(0)>0$ ) for all delays $\tau_{i j}$ satisfying (1.2) if and only if $a_{i i}<0$ for $i=1, \ldots, n$, $\operatorname{det} A \neq 0$ and $A$ is weakly diagonally dominant.

Proof. For the necessity part, first we observe that in the course of proving Theorem 1 , we have shown that, under the assumption $\operatorname{det}(A) \neq 0$, the characteristic equation (2.1) has a root with positive real part (for a certain choice of $\tau_{i j}$ satisfying (1.2)), provided $a_{i i} \geq 0$ for some $i$ or $A$ is not weakly diagonally dominant. Next, let $B=\left(\hat{y}_{i} a_{i j}\right)$ be the linearization of $(3.1)$ at $\hat{y}$. Then $\operatorname{det}(B) \neq 0$, because otherwise $\operatorname{det}(A)=0$ and (3.1) has many equilibrium solutions near $\hat{y}$ (implying $\hat{y}$ is not asymptotically stable). Now, since $\hat{y}$ is asymptotically stable, none of the roots of (2.1), with $A$ replaced by $B$, can have positive real parts (cf. Theorem 2.1 of Hale and Verduyn Lunel [7 p. 314]). Hence, $b_{i i}<0$ for all $i$ and $B$ is weakly diagonally dominant. This implies $a_{i i}<0$ for all $i$ and $A$ is weakly diagonally dominant as well.

For the sufficiency part, the proof in section 2 only gives (local) asymptotic stability. When $A$ is irreducible, it turns out that one can choose $\alpha_{i}>0$ and $\beta_{i j}>0$ appropriately (see later) so that

$$
V(y(.), t)=\sum_{i=1}^{n} \alpha_{i}\left(y_{i}(t)-\hat{y}_{i} \log y_{i}(t)\right)+\sum_{i, j=1}^{n} \beta_{i j} \int_{t-\tau_{i j}}^{t}\left(y_{j}(s)-\hat{y}_{j}\right)^{2} d s
$$

becomes a Lyapunov functional. For the case of a general $A$, we will use induction to show that every solution $y(t)$ of (3.1) with positive initial conditions converges to $\hat{y}$.

Denoting $y-\hat{y}$ by $x,(3.1)$ can be written as

$$
\dot{y}_{i}(t)=y_{i}(t) \sum_{j=1}^{n} a_{i j} x_{j}\left(t-\tau_{i j}\right)
$$

The derivative of $V$ along a solution $y(t)$ of (3.1) is then given by

$$
\dot{V}=\sum_{i, j=1}^{n} \alpha_{i} x_{i}(t) a_{i j} x_{j}\left(t-\tau_{i j}\right)+\sum_{i, j=1}^{n} \beta_{i j}\left[x_{j}^{2}(t)-x_{j}^{2}\left(t-\tau_{i j}\right)\right] .
$$

First, we assume that $A$ is an irreducible weakly diagonally dominant matrix. Then there exist $c_{i}>0$ such that $(2.2)$ holds. Moreover, there also exist $d_{i}>0$ such that

$$
d_{i} a_{i i}+\sum_{j \neq i} d_{j}\left|a_{j i}\right| \leq 0 \quad \text { for } i=1, \ldots, n .
$$

(The vectors $c$ and $d$ may be chosen as right and left eigenvectors of $\tilde{A}$.)

By choosing $\alpha_{i}=2 \frac{d_{i}}{c_{i}}$ and $\beta_{i j}=\frac{d_{i}}{c_{j}}\left|a_{i j}\right|$, we have

$$
\dot{V}=\sum_{i \neq j} \frac{d_{i}}{c_{j}}\left|a_{i j}\right| x_{j}^{2}(t)+2 \sum_{i, j=1}^{n} \frac{d_{i}}{c_{i}} a_{i j} x_{i}(t) x_{j}\left(t-\tau_{i j}\right)-\sum_{i \neq j} \frac{d_{i}}{c_{j}}\left|a_{i j}\right| x_{j}^{2}\left(t-\tau_{i j}\right) .
$$


After collecting terms, the coefficient of $x_{i}^{2}(t)$ (for $i$ fixed) is given by

$$
\sum_{j=1, j \neq i}^{n} \frac{d_{j}}{c_{i}}\left|a_{j i}\right|+2 \frac{d_{i}}{c_{i}} a_{i i}
$$

By adding $\frac{1}{c_{i}}$ times (3.5) to $\frac{d_{i}}{c_{i}^{2}}$ times (2.2), we see that this coefficient is no larger than $-\sum_{j=1, j \neq i}^{n} \frac{d_{i} c_{j}}{c_{i}^{2}}\left|a_{i j}\right|$. Hence (3.6) reduces to

$$
\dot{V} \leq-\sum_{i \neq j} \frac{d_{i}}{c_{j}}\left|a_{i j}\right|\left(\frac{c_{j}}{c_{i}} x_{i}(t) \operatorname{sgn} a_{i j}-x_{j}\left(t-\tau_{i j}\right)\right)^{2} \leq 0 .
$$

The boundedness of $V$ along a positive solution $y(t)$ implies the existence of constants $m, M>0$ such that $m \leq y_{i}(t) \leq M$ for all $i$ and for all $t \geq 0$. Hence forward orbits are precompact and the $\omega$-limit set exists and is nonempty, compact and invariant (cf. Lemma 1.3 of Hale and Verduyn Lunel [7. p. 103]). By LaSalle's theorem, $\omega(y()$.$) is contained in the maximal compact invariant subset of \dot{V}=0$.

Equality $\dot{V} \equiv 0$ is possible for $y(.) \not \equiv \hat{y}$ only if there is equality in (2.2) and (3.5) (i.e., $\operatorname{det} \tilde{A}=0)$ and $x_{j}\left(t-\tau_{i j}\right)=\frac{c_{j}}{c_{i}} x_{i}(t) \operatorname{sgn} a_{i j}$ holds for all $t$ and all $i, j$ with $i \neq j$ and $a_{i j} \neq 0$. Inserting this into (3.1), we obtain

$$
\dot{y}_{i}=y_{i}\left[a_{i i} x_{i}(t)+\sum_{j=1, j \neq i}^{n} a_{i j} x_{j}\left(t-\tau_{i j}\right)\right]=y_{i}\left[a_{i i} x_{i}(t)+\sum_{j=1, j \neq i}^{n}\left|a_{i j}\right| \frac{c_{j}}{c_{i}} x_{i}(t)\right]=0
$$

Hence $y(t)$ must be a constant solution of the differential equation (3.1). Since $\operatorname{det} A \neq 0$ by assumption, $x(t) \equiv 0$, i.e. $y(t) \equiv \hat{y}$ is the only positive constant solution of (3.1). Thus $\{\hat{y}\}$ is the global attractor and hence the maximal compact invariant subset in $C\left([-\tau, 0]\right.$, int $\left.\mathbb{R}_{+}^{n}\right)$, the space of positive continuous functions on $[-\tau, 0]$, where $\tau=\max _{i, j}\left\{\tau_{i, j}\right\}$. In particular, every positive solution $y(t)$ of (3.1) converges to $\hat{y}$ as $t \rightarrow \infty$, when $A$ is irreducible.

Next, we consider the case of a general $A$. We will use induction on $n$ to show that (i) for any positive solution $y(t)(t \geq 0)$ of (3.1), there exist $0<m \leq M$ such that $m \leq y_{i}(t) \leq M$ for all $t \geq 0$ and $i$, and that $\lim _{t \rightarrow \infty} y(t)=\hat{y}$ and (ii) if $y(t)$ is a positive solution (3.1) defined for all $t \in \mathbb{R}$ such that $m \leq y_{i}(t) \leq M$ for all $t$, where $0<m \leq M$, then $y(t) \equiv \hat{y}$.

If $A$ is irreducible, then the assertions hold by the above Lyapunov functional argument. So we assume $A$ is reducible. By a renumbering of indices, $A$ can be written in block triangular form $A=\left(\begin{array}{cc}A_{11} & A_{12} \\ O & A_{22}\end{array}\right)$, with $A_{11}$ irreducible of order $k$. The delay equation (3.1) can then be written schematically as a $2 \times 2$ system

$$
\begin{aligned}
& \dot{Y}_{1}=Y_{1}\left(A_{11} X_{1}+A_{12} X_{2}\right), \\
& \dot{Y}_{2}=Y_{2} A_{22} X_{2} .
\end{aligned}
$$

Since the order $n-k$ of $A_{22}$ is strictly less than that of $A$, by the induction hypothesis on the second half of (3.8), we can assume that $y_{i}(t)$ remains bounded (both from 0 and $\infty)$ for all $i=k+1, \ldots, n$ and $t \geq 0$ and $Y_{2}(t) \rightarrow \hat{Y}_{2}=\left(\hat{y}_{k+1}, \ldots, \hat{y}_{n}\right)$ as $t \rightarrow \infty$. From the linear analysis of section 2 , we can further infer that $\left|X_{2}(t)\right| \leq C e^{-\varepsilon t}$ $(t \geq 0)$ for suitable constants $C, \varepsilon>0$. 
Now consider again the function $V$ from (3.3), with $n$ replaced by $k$, the size of the irreducible block $A_{11}$. Then a similar computation, taking into account (3.7) for the terms arising from $A_{11}$, leads to (with $\left|X_{1}\right|$ denoting the norm of the vector $\left.X_{1}=\left(x_{1}, \ldots x_{k}\right)\right)$

$$
\begin{aligned}
\dot{V} & \leq \sum_{i=1}^{k} \alpha_{i} x_{i}(t)\left[\sum_{j=k+1}^{n} a_{i j} x_{j}\left(t-\tau_{i j}\right)\right] \\
& \leq C_{1}\left|X_{1}(t)\right| e^{-\varepsilon t} \leq C_{2}\left(V(t)+C_{3}\right) e^{-\varepsilon t}
\end{aligned}
$$

This differential inequality shows that $V(t)$ stays bounded as $t \rightarrow \infty$; and hence the first $k$ components of $y(t)$ stay bounded (both from 0 and from $\infty$ ). This shows that there exist $0<m \leq M$ such that $m \leq y_{i}(t) \leq M$ for all $i=1, \ldots, n$ and $t \geq 0$.

Now consider a (full) solution $y(t)=\left(Y_{1}(t), Y_{2}(t)\right)$ of $(3.1)$ defined for all $t \in \mathbb{R}$ such that $m \leq y_{i}(t) \leq M$ for all $i$ and $t$, where $0<m \leq M$. Applying the induction hypothesis to the second part of (3.8), we deduce that $Y_{2}(t) \equiv \hat{Y}_{2}$. Thus $X_{2}(t) \equiv 0$. Hence $Y_{1}(t)$ satisfies $\dot{Y}_{1}=Y_{1} A_{11} X_{1}$. Since $A_{11}$ is irreducble, we conclude $Y_{1}(t) \equiv \hat{Y}_{1}$. Hence $y(t) \equiv \hat{y}$.

Lastly, let $y(t)(t \geq 0)$ be a positive solution of (3.1). Pick any $z_{t}$, a full orbit in $\omega\left(y_{t}\right)$, the $\omega$-limit set of the orbit $y_{t}$. Then $z(t)$ is a solution of (3.1) defined for all $t \in \mathbb{R}$ which is bounded (from 0 and $\infty$ ). As was shown in the previous paragraph, $z(t) \equiv \hat{y}$. Hence $\omega\left(y_{t}\right)=\{\hat{y}\}$ and $\lim _{t \rightarrow \infty} y(t)=\hat{y}$, which completes the induction proof.

Remark. We note that the sufficiency part for the linear case (Theorem 1) can also be shown by basically the same argument as above, using the Lyapunov function $V(x(), t)=.\sum_{i=1}^{n} \alpha_{i} x_{i}^{2}(t)+\sum_{i, j=1}^{n} \beta_{i j} \int_{t-\tau_{i j}}^{t} x_{j}^{2}(s) d s$ instead of (3.3).

\section{REFERENCES}

[1] L. V. Ahlfors, Complex analysis: an introduction to the theory of analytic functions of one complex variable (3rd ed.), McGraw-Hill, New York, 1979. MR 80c:30001

[2] A. Berman and R. Plemmons, Nonnegative matrices in the mathematical sciences, SIAM, Philadelphia, 1994. MR 95e:15013

[3] L. E. El'sgol'ts and S.B. Norkin, Introduction to the theory and application of differential equations with deviating arguments, Academic Press, New York, 1973. MR 50:5134

[4] M. Fiedler, Special matrices and their applications in numerical mathematics, Martinus Nijhoff Publ. (Kluwer), Dordrecht, 1986. MR 92b:15003

[5] K. Gopalsamy, Harmless delays in a periodic ecosystem, J. Austral. Math. Soc. B 25 (1984), 349-365. MR 85c:92031

[6] I. Győri, Stability in a class of integrodifferential systems, Agarwal, R. P. (ed.), Recent trends in differential equations., World Sci. Ser. Appl. Anal. 1, Singapore: World Scientific Publishing., 1992, pp. 269-284. MR 93e:34005

[7] J. K. Hale and S.M. Verduyn Lunel, Introduction to functional differential equations, Springer-Verlag, New York, 1993. MR 94m:34169

[8] J. Hofbauer, K. Sigmund, The theory of evolution and dynamical systems, Cambridge University Press, Cambridge, 1988. MR 91h:92019

[9] Y. Kuang, Delay Differential equations with applications in population dynamics, Academic Press, New York, 1993. MR 94f:34001

[10] P. Lancaster and M. Tismenetsky, The theory of matrices (2nd ed.), Academic Press, New York, 1985. MR 87a:15001]

[11] Z. Lu and Y. Takeuchi, Global dynamic behavior for Lotka-Volterra systems with a reducible interaction matrix, J. Math. Anal. Appl. 193 (1995), 559-572. MR 96f:34071 
[12] Z. Lu and W. Wang, Global stability for two-species Lotka-Volterra systems with delay, J. Math. Anal. Appl. 208 (1997), 277-280. MR 97m:34152

[13] R. Redheffer, A new class of Volterra differential equations for which the solutions are globally asymptotically stable, J. Diff. Equations 82 (1989), 251-268. MR 91f:34058

Institut für Mathematik, Universität Wien, A-1090 Wien, Austria

E-mail address: Josef.Hofbauer@univie.ac.at

Department of Mathematical Sciences, University of Alberta, Edmonton, Alberta, Canada T6G 2G1

E-mail address: joseph.so@ualberta.ca 